(VLCD) help in weight reduction and are known to shrink the liver. Our aim was to assess the role of ARFI in assessing and monitoring the change in liver architecture in a cohort of morbidly obese patients in response to VLCD.

Methods A cohort of non-diabetic morbidly obese patients at risk for NASH was selected for this study (clinical trial no: NCT01950052). Liver volume was estimated with the help of a standardised ultrasound protocol while liver fibrosis was analysed with ARFI. After randomisation, a very low calorie diet (800 kcal) was given to one group while the rest were controls. Four weeks later, ARFI was repeated and all patients underwent a laparoscopic roux-en-y gastric bypass. A liver biopsy was taken during surgery from the same liver segment as the ARFI measurements. The liver histology was evaluated according to the NASH Clinical Research Network Scoring System by two blinded pathologists. Steatosis, fibrosis and NAFLD activity scores were correlated with ARFI scores.

Results Liver volume shrank by $21.5 \%$ in the diet arm $(\mathrm{n}=10)$ compared to $2 \%(\mathrm{p}<0.05)$ in the control arm $(\mathrm{n}=14)$ in 4 weeks. The ARFI scores were similar in the diet and control group [median $2.92(1.1-3.8) \mathrm{m} / \mathrm{s}$ vs. $3.22(1.54-3.65) \mathrm{m} / \mathrm{s}, \mathrm{p}=$ $0.7], \mathrm{p}=0.7]$ at recruitment and at the time of the biopsy 4 weeks later [2.16 (1.19-3.68) m/s vs. $2.83(1.5-3.48) \mathrm{m} / \mathrm{s}, \mathrm{p}=$ $0.3]$. ARFI demonstrated a drop in values in the diet group $(\mathrm{p}=$ 0.1 ) but this was not significant. Similarly, liver biopsy at surgery confirmed a trend of lower levels of steatosis in the diet group (27 vs. $42 \%, p=0.12$ ). The ARFI scores did not correlate with the steatosis grade $(\mathrm{p}=0.8)$, or NAFLD score $(\mathrm{p}=0.48)$.

Conclusion Low calorie diets shrink liver volumes but ARFI could not detect any change in liver stiffness. ARFI does not appear to correlate with liver steatosis and may not be ideally suited for short term monitoring of successful treatment of NASH. However its role in long term monitoring needs further evaluation.

Disclosure of Interest None Declared.

\section{PTH-089 HEPATITIS B MONOTHERAPY WITH TENOFOVIR OR ENTACAVIR: A UK SINGLE CENTRE EXPERIENCE}

S Shabbir*, S Stevenson, PM Tachtatzis, LC Claridge, MH Davies, RL Jones, MA Aldersley. Hepatology, St James's University Hospital, Leeds, UK

\subsection{6/gutjnl-2014-307263.535}

Introduction Tenofovir (TDF) and Entacavir (ETV) are potent oral antiviral medication for chronic hepatitis $\mathrm{B}{ }^{1}$. Our aim was to record effectiveness of these 2 widely used antivirals in achieving viral suppression, $\mathrm{HBeAg}$ seroconversion and $\mathrm{HBsAg}$ loss after an initial 12 months of treatment and to further assess long term results (from June 2007 to April 2013) in chronic hepatitis B patients in a single tertiary referral centre.

Methods We retrospectively collected data from hospital record from June 2007 to April 2013. We included chronic hepatitis B patients with high viral load $(>2000 \mathrm{IU} / \mathrm{ml})$, treatment naive and treatment experienced and who were on treatment for at least 12 months with either on tenofovir or entacavir. Treatment experienced patients were those who were switched from other antivirals to tenofovir or entacavir with high viral load.

Results 61 patients were treated with TDF monotherapy for a median of 29 months, 25 (41\%) were $\mathrm{HBeAg}$ positive and 36 (59\%) were $\mathrm{HBeAg}$ negative. In the $\mathrm{HBsAg}$ positive group 17 $(68 \%)$ achieved virological response in 12 months time while 22 $(88 \%)$ had achieved it on longer term treatment. $2(8 \%)$ got HBeAg seroconvertion within 12 months whilst 4 (16\%) seroconverted on longer term treatment. In the $\mathrm{HBeAg}$ negative group $29(81 \%)$ achieved virological response after 12 months treatment whilst 34 (94\%) achieved virological response on longer term treatment.

33 patients were treated with ETV monotherapy for a median of 38 months, 14 (42\%) were $\mathrm{HBeAg}$ positive and 19(58\%) were $\mathrm{HBeAg}$ negative. In the $\mathrm{HBsAg}$ positive group 7 (50\%) achieved virological response after 12 months treatment whilst all $14(100 \%)$ achieved virological response on longer term treatment. $1(7 \%)$ got $\mathrm{HBeAg}$ seroconvertion in 12 months whilst $4(29 \%)$ seroconverted on longer term treatment. In the HBeAg negative group 15 (79\%) achieved virological response after 12 months time whilst all 19 (100\%) had achieved it on longer term treatment.

None of the patients on either on ETV or TDF lost HBsAg. Conclusion ETV and TDF are potent nucleos ( $t$ )ide analogues as first-line monotherapies for chronic hepatitis B. Due to the fact that ETV was licensed before TDF treatment durations are longer with this agent which is likely to explain the numerically superior long term results with ETV. It will require more patients and longer duration of treatment to allow a meaningful comparison of the two agents and to determine if HBsAg loss as described in the registration trials can be replicated in clinical practice.

\section{REFERENCE}

1 Woo G, Tomlinson G, Nishikawa $Y$, et al. Tenofovir and entecavir are the most effective antiviral agents for chronic hepatitis B: a systematic review and Bayesian meta-analyses. Gastroenterology. 2010 Oct;139(4):1218-29

Disclosure of Interest None Declared.

\section{PTH-090 NATURAL HISTORY OF NAFLD: A STUDY OF 108 PATIENTS WITH PAIRED LIVER BIOPSIES}

1,2S Mcpherson*, 'E Henderson, ${ }^{2} \mathrm{~T}$ Hardy, ${ }^{3} \mathrm{~A}$ Burt, ${ }^{1,2} \mathrm{C}$ Day, ${ }^{1,2} \mathrm{QM}$ Anstee. ${ }^{1}$ Liver Unit, Freeman Hospital, Newcastle Upon Tyne, UK; ${ }^{2}$ Institute of Cellular Medicine, Newcastle University, Newcastle Upon Tyne, UK; ${ }^{3}$ School of Medicine, University of Adelaide, Adelaide, Australia

\subsection{6/gutjnl-2014-307263.536}

Introduction Non-alcoholic fatty liver disease (NAFLD) is the most common cause of liver disease in many countries. There remains considerable uncertainty about natural history and prognosis. Few studies, totalling $<400$ patients, have examined the evolution of steatosis/steatohepatitis and fibrosis of NAFLD in patients with paired biopsies. In general it is thought that fibrosis progression in patients with "NAFL" (steatosis +/- mild inflammation) is uncommon, whereas non-alcoholic steatohepatitis (NASH; steatosis + hepatocyte ballooning and inflammation) more frequently progresses. Our aim was to assess the histological severity of NAFLD in a cohort with serial liver biopsy data and to determine clinical factors that predict fibrosis progression.

Methods Patients with 2 liver biopsies $>1$ year apart were identified from the Newcastle Hospitals NAFLD clinic. Clinical and laboratory data were collected from the time of liver biopsy.

Results 108 patients (mean age $48 \pm 12$ years; 66\% male; $48 \%$ diabetic) were identified with $\geq 2$ liver biopsies (median interval 6.6 years, range 1.3-22.6). 81 (75\%) patients had NASH and 27 patients with NAFL. Overall 45 (42\%) patients had progression of fibrosis, 43 (40\%) had no change in fibrosis, while $20(18 \%)$ had fibrosis regression. The mean rate of fibrosis was $0.08 \pm$ 0.25 stages/year overall, increasing to $0.29 \pm 0.24$ stages/year in 